\title{
Integrative analysis of microRNA and mRNA expression profiles in fetal rat model with anorectal malformation
}

\author{
Hui Xiao ${ }^{1,2}{ }^{\text {, Rui Huang }}{ }^{3}{ }^{,}$Mei Diao ${ }^{1}$, Long $\mathbf{L i}^{1}{ }^{\text {, Dai Xiao Cui }}{ }^{\text {Corresp. }} 3$ \\ 1 Paediatric surgery, Capital institute of Pediatric, Beiling, China \\ 2 Graduate School of Peking Union Medical College, Chinese Academy of Sciences, Beijing, China \\ 3 Department of Key Laboratory, Capital Institute of Paediatric, Beijing, China \\ Corresponding Author: Dai Xiao Cui \\ Email address: xiaodai_cui@sina.com
}

\begin{abstract}
Background Anorectal malformations (ARMs) are the most common congenital malformation of the gut, and regulated by multiple signal transduction pathways. The microRNA (miRNA) expression profiles and their biologial functions in anorectal malformations (ARMs) remain unclear. The aim of our study was to evaluate miRNA and mRNA expression profiles in the ARM rats. Methods and materials ARM was induced with ethylenethiourea (ETU) on gestational day 10. Cesarean deliveries were performed to harvest the embryos on gestional gay 20 . For the extraction of total RNA, $1 \mathrm{~cm}$ termianl hindgut samples were obtained from three fetal rats that had similar weights. The microarrays and quantitative RT-PCR analysis were conducted to evaluate the miRNA and mRNA expression profiles in normal fetal rats $(n=3)$ and ARM fetal rats $(n=3$ ). Results In total, 33 miRNAs and 772 mRNAs were significantly and differentially expressed in termianl hindgut tissues of ARM group versus control group. Functional annotation was performed to understand the functions and pathways of differentially expressed mRNAs. And we constructed a miRNA-target gene regulatory network including 25 differentially expressed miRNAs and 76 mRNAs. Furthermore, the credibility of the microarray-based results were validated by using qRT-PCR.Conclusion The miRNA and mRNA expression in terminal hindgut tissue in ARM fetal rat might provide a basis for further research on the pathogenesis of ARMs
\end{abstract}


1 Title: Integrative analysis of microRNA and mRNA expression profiles in fetal rat model with

2 anorectal malformation

3 Running title: microRNAs expression profiles in Anorectal Malformations

4 Author names: Hui Xiao, $\mathrm{MD}^{1,2}$, Rui Huang, $\mathrm{MD}^{3}$, Mei Diao, MD ${ }^{1}$, Long Li, MD ${ }^{1}$, Dai 5 Xiao_Cui, MD ${ }^{3 *}$

7 The affiliation and address of the authors:

$8{ }^{1}$ Department of Pediatric Surgery, Capital Institute of Pediatrics, Beijing, People's Republic of 9 China

$10{ }^{2}$ Graduate School of Peking Union Medical College, Chinese Academy of Medical Sciences,

11 Beijing, People's Republic of China

$12{ }^{3}$ Department of Key Laboratory, Capital Institute of Pediatrics, Beijing, People's Republic of 13 China

\section{*Corresponding author:}

Dai Xiao_Cui, Email: xiaodai_cui@sina.com, Telephone: +8615811157596

\section{First author:}

18 Hui Xiao, E-mail: huixiao1989@163.com

\section{Other authors:}

20 Rui Huang, E-mail: huangrui_alice@126.com

21 Mei Diao, E-mail: diaomei2017@sina.com 
22 Long Li, E-mail: 849993127@qq.com

23 Words count: 4691 (text, excluding abstract, acknowledgment and references)

24 Tables count: 2

25 Figures count: 6

26 Acknowledgment: None

27 Corresponding Author: Dai Xiao_Cui, Email: xiaodai_cui@sina.com, Telephone:

28 +8615811157596. Department of Key Laboratory, Capital Institute of Pediatrics, Beijing,

29 People's Republic of China

30

31

32

33

34

35

36

37

38

39

40

41 

rat model with anorectal malformation

\section{ABSTRACT}

Background Anorectal malformations (ARMs) are the most common congenital malformation of the gut, and regulated by multiple signal transduction pathways. The microRNA (miRNA) expression profiles and their biologial functions in anorectal malformations (ARMs) remain unclear. The aim of our study was to evaluate miRNA and mRNA expression profiles in the ARM rats.

Methods and materials ARM was induced with ethylenethiourea (ETU) on gestational day 10. Cesarean deliveries were performed to harvest the embryos on gestional day 20. For the extraction of total RNA, $1 \mathrm{~cm}$ terminal hindgut samples were obtained from three fetal rats that had similar weights. The microarrays and quantitative RT-PCR analysis were conducted to evaluate the miRNA and mRNA expression profiles in normal fetal rats $(n=3)$ and ARM fetal rats $(n=3)$.

Results In total, 33 miRNAs and 772 mRNAs were significantly and differentially expressed in termianl hindgut tissues of ARM group versus control group. Functional annotation was performed to understand the functions and pathways of differentially expressed mRNAs. And we constructed a miRNA-target gene regulatory network including 25 differentially expressed 
miRNAs and 76 mRNAs. Furthermore, the credibility of the microarray-based results were validated by using qRT-PCR.

Conclusion The miRNA and mRNA expression in terminal hindgut tissue in ARM fetal rat might provide a basis for further research on the pathogenesis of ARMs.

\section{Introduction}

Anorectal malformations (ARMs) are among the most common congenital abnormalities with a wide clinical spectrum, occuring in approximately 1/5000-1/1500 live births (Endo $M$ et al., 1999; Van der Putte SC, 1986; Tsuda T et al., 2011; Levitt MA et al., 2005). Rencent studies demonstrated that ARMs are a group of complex malformations affected by many factors, and genetic factors has a great influence on the pathogenesis of ARM, such as Shh, Wnt, BMP-4, Gli2, Hox, Fgf, PCSK5, P63, and others (Wang C et al., 2015; Wong EH et al., 2013; Khanna K et al., 2018). Although ARM has been extensively studied, the detailed pathogenesis of ARM is still unclear, especially the molecular mechanisms that involved in the ARMs remain uncharacteristic. Non-coding RNAs (ncRNAs), it is generally known that, are a family of RNA molecules that regulate gene expression but do not code proteins typically, ncRNAs participate in a variety of cellular processes including cell metabolic function and development (Mattick JS et al., 2006; Thum T et al., 2015; Godwin JG., 2010; Wang KC et al., 2011). NcRNAs can be subdivided into long ncRNAs (lncRNAs) with a length range from 0.2 and $2 \mathrm{~Kb}$ and small ncRNAs ( $<200$ nucleotides long), which encompass microRNAs (miRNAs). In eukaryotic cells, miRNAs can regulate $31 \%$ of the target genes and participate in multiple processes of biological 
84

85

development and diseases (Lewis BP et al., 2013). However, there is paucity of literatures concerning on the analysis of miRNA and mRNA co-expression in the development of ARMs. Hence the purpose of our study was to integrate miRNA-mRNA expression profiles in the terminal hindgut tissue of ARM fetal rats at twentieth day of embryo (E20) with using Agilents miRNA and mRNA microarrays. In addition, the relevant data were used for bioinformatics analysis, which includes predicted target genes, GO enrichment analysis, and KEGG pathway analysis.

\section{Materials and methods}

\subsection{Animals}

Ethical approval was obtained from the Capital Institute of Pediatric prior to the study. Totally, 18 healthy and mature SD of similar weight (12 females, 6 males) were provided by the Beijing Vital River Laboratory Animal Technology Co,. Ltd. The procedures for generating ARMs in fetal rats are described in earlier reports (Bai YZ et al., 2004). The rats were divided into two equal groups randomly. Two female rats were mated to one male rat. The presence of sperm in a vaginal smear was deemed to be as Gestational day 0 after overnight mating. The pregnant rats were divided into experiment group and control group randomly. In the experimental group, 6 pregnant rats received a single dose of 1\% ethylenethiourea (ETU, 125 $\mathrm{mg} / \mathrm{kg}$ ) on gestational day 10. In the control group, the 6 pregnant rats received an corresponding amount of distilled water on gestational day 10. Embryos were acquired by cesarean delivery on GD 20. The presence of ARMs was identified through the light microscope (please 
105

106

107

108

109

110

111

112

113

114

115

116

117

118

119

120

121

122

123

124

125

supplementary material 1), then the embryos were subdivided into ARM group and normal group. On gestational day 20, 12 pregnant rats of similar weight were acquired by cesarean section, thereby resulting in approximately 100 fetal rats in total. One centimeter of terminal hindgut tissue from the embryos was resected, and frozen in liquid nitrogen immediately to prepare for the extraction of RNA with Trizol methods.

\subsection{RNA isolation and quantification}

Total RNA was isolated with the TRIzol reagent (Invitrogen) according to the manufacturer's protocol. Total RNA was quantified by the NanoDrop ND-2000 (Thermo Scientific) and the RNA integrity was assessed with using Agilent Bioanalyzer 2100 (Agilent Technologies, Agilent Bioanalyzer 2100 inclusion criteria: A: RIN $\geq 7$ and 28S/18S $\geq 0.7$, follow-up experiments can be carried out). The sample labeling, microarray hybridization, and washing were performed based on the manufacturer's standard protocols. Briefly, 5ug total RNA were dephosphorylated, denaturated and then labeled with Cyanine-3-CTP. After purification the labeled RNAs were hybridized onto the microarray. RNA quantification detail was described in

\section{supplementary material 2.}

\subsection{Microarray analysis}

Three ETU-induced ARM fetal rats and three control rats were chosen for microarray analysis randomly. The Agilent Rat miRNA Microarray $\left(8^{*} 15 \mathrm{~K}\right)$ and Agilent Rat lncRNA V2 $(8 * 60 \mathrm{~K})$ microarray (including mRNA microarray) were utilized to evaluate miRNA and mRNA expression profiles, respectively, in the two groups. Microarray analyses were performed by Shanghai OEBiotech.Co., Ltd, Shang hai, China. 
126

127

128

129

130

131

132

133

134

135

136

137

138

139

140

141

142

143

144

145

146

\subsection{Real-time RT-PCR of miRNA and mRNA}

The process of Real-time RT-PCR of miRNA were as follows: each PCR reaction contained $3 \mu \mathrm{l}$ cDNA, $14 \mu \mathrm{l}$ mQ- $\mathrm{H}_{2} \mathrm{O}, 3 \mu \mathrm{l}$ PCR-buffer, $3 \mu \mathrm{dNTP}$ solution, $3 \mu \mathrm{l}$ BSA solution, $1 \mu \mathrm{T}$ Taqpolymerase, $0.17 \mu \mathrm{l}$ uracil-DNA glycosylase, and $3 \mu \mathrm{l}$ of corresponding primer. The reactions were performed under the following conditions: initial denaturation at $94{ }^{\circ} \mathrm{C}$ for $2 \mathrm{~min}, 50$ cycles; denaturation at $94{ }^{\circ} \mathrm{C}$ for $10 \mathrm{~s}$; and annealing and elongation at $60{ }^{\circ} \mathrm{C}$ for $20 \mathrm{~s}$. PCR specificity was controlled by melting curves. In each experiment, one plate contained samples of analyzed cDNA with a primer for the target genes and the reference gene (3 repeats each). The relative levels of gene expression were determined as $\Delta \mathrm{Ct}=\mathrm{Ct}$ gene- $\mathrm{Ct}$ reference, and the fold change in gene expression was calculated with the $2-\Delta \Delta^{\mathrm{Ct}}$ method. Experiments were repeated in triplicate.

The process of Real-time RT-PCR of mRNA were as follows: RNA ( $1 \mu \mathrm{g})$ was reversetranscribed by using the QuantiTect Reverse Transcription Kit (Qiagen) following the manufacturer's instructions. Each Quantitative real-time PCR was performed using $0.3 \mu l$ of cDNA in a final volume of $25 \mu \mathrm{l}$ under the following conditions: $50{ }^{\circ} \mathrm{C}$ for $2 \mathrm{~min}, 95{ }^{\circ} \mathrm{C}$ for 10 min, 40 cycles of $95{ }^{\circ} \mathrm{C}$ for $15 \mathrm{~s}, 60{ }^{\circ} \mathrm{C}$ for $60 \mathrm{~s}$. A dissociation procedure was performed to generate a melting curve for confirmation of amplification specificity. GAPDH was used as the reference gene. The relative levels of gene expression were determined as $\Delta \mathrm{Ct}=\mathrm{Ct}$ gene $-\mathrm{Ct}$ reference, and the fold change in gene expression was calculated with the $2-\Delta \Delta^{\mathrm{Ct}}$ method. Experiments were repeated in triplicate. The primers sequences for miRNAs and mRNAs can be seen in supplementary material.3

\subsection{Target prediction and function analysis}



utilized to predict the potential target genes. The Target Scan version 7.1 was used to obtain the predicted mRNA targets for each differential expression of miRNA. Then these predicted genes were compared with the mRNA microarray datas, and overlaps of them were determined. The Gene Ontology and KEGG Pathways were considered to elucidate the biological functions of these differentially expressed miRNA target genes, and the cytoscape was adopted to conduct the network analysis.

\section{Statistical analysis}

The SPSS version 21.0 package (Chicago, IL, USA) was used for statistical analysis. The independent sample student $t$ test, analysis of variance, and nonparametric test were rationally utilized for the between-group differences. The $P$ value $<0.05$ was indicated to be significant difference.

\section{Results}

\section{1. miRNA and mRNA differential expression profiles}

The miRNA expression profiles were significantly different in terminal hindgut tissues

between the ARM and control groups. Process of the microarray and data analysis was shown as

follows (Fig 1). Of all the miRNA measured, 33 miRNAs were identified. Among the differently expressed miRNAs, 8 miRNAs were up-regulated in the terminal hindgut tissue of the ARM group, while 25 miRNAs were down-regulated ( $>2$-fold changes, $\mathrm{P}<0.05$; Table. 1). The cluster analysis was shown in Fig. 2. In order to investigate the potential targets of the altered 
168

169

170

171

172

173

174

175

176

177

178

179

180

181

182

183

184

185

186

187

188

miRNAs, we also determined the mRNA expression profiles of the terminal hindgut tissues by using Agilent microarray. Greater than 20,000 rat genes and transcripts were investigated and the ARM group and control group were compared. Among the 772 differentially expressed mRNAs, 350 of the genes were significantly up-regulated and 422 were down-regulated $(\geq 2.0$ fold changes, $\mathrm{P}<0.05)$ in the ARM group compared with control group. The top 20 upregulated genes and the top 20 downregulated genes were listed in Table. 2.

\subsection{GO and KEGG pathway analysis of the predicted miRNA targets}

The Gene Ontology (GO) system was used for functional enrichment analysis of the differentially expressed miRNAs predicted target genes. GO functional annotation of the predicted target genes of the differentially expressed miRNAs was shown in Fig. 3A. According to molecular function analysis, there were $166 \mathrm{GO}$ terms significantly enriched $(\mathrm{P}<0.05)$, and they were predominantly related to protein binding, identical protein binding, etc. in ARM development process. Analysis of the biological processes revealed the 622 significantly enriched GO terms $(\mathrm{P}<0.05)$ to be predominantly associated with transcription, DNA-templated in ARM development process. Cellular component analysis identified 143 significantly enriched GO terms $(\mathrm{P}<0.05)$ to be primarily related to cytoplasm, extracellular exosome in ARM.

The potential functional pathways of differentially expressed miRNA targets were then predicted by KEGG pathway system. The DAVID, and KEEG pathway system was used to analysis the microarray data. It showed that 64 pathways were predicted to be significantly related to ARM $(\mathrm{P}<0.05)$. HIF-1 signaling pathway, Cytokine-cytokine receptor interaction, Pathways in cancer, GABAergic synapse, and cAMP signaling pathway were the main pathways 
189

190

191

192

193

194

195

196

197

198

199

200

201

202

203

204

205

206

207

208

209

involved in these predicted pathways (Fig. 3B).

\subsection{GO and KEGG pathway analysis of the differentially expressed mRNAs}

The Gene Ontology (GO) system was used for functional enrichment analysis of the differentially expressed mRNAs. The top $10 \mathrm{GO}$ pathway enrichment terms for differentially expressed mRNAs were shown in Fig.4A. According to molecular function analysis, there were 68 GO terms significantly enriched $(\mathrm{P}<0.05)$, and they were closely related to heparin binding, actin binding, tropomyosin binding, etc. in ARM development process. According to biological processes analysis, it revealed that 331 significantly enriched GO terms $(\mathrm{P}<0.05)$ to be predominantly associated with muscle contraction, muscle organ development, cardiac muscle contraction, and regulation of muscle contraction in ARM development process. Cellular component analysis identified 56 significantly enriched GO terms $(\mathrm{P}<0.05)$ to be primarily related to extracellular space, myofibril, and troponin complex in ARM.

The KEGG pathways for the differentially expressed mRNAs were then performed by KEGG pathway system. The DAVID and KEEG pathway system were used to analysis microarray data. It revealed that 53 pathways were predicted to be significantly related to ARM $(\mathrm{P}<0.05)$. Dilated cardiomyopathy, Hypertrophic cardiomyopathy $(\mathrm{HCM})$, Cell adhesion molecules (CAMs), and ECM-receptor interaction were the main pathways involved in the development process of ARM (Fig. 4B).

\section{4. miRNA target gene prediction}

As miRNAs negatively regulate mRNAs expression via degradation or translation inhibition. We intersect the predicted target mRNAs of upregulated miRNAs with the downregulated 
210

211

212

213

214

215

216

217

218

219

220

221

222

223

224

225

226

227

228

229

230

mRNAs from microarray, and the predicted target of mRNAs of downregulated miRNAs with the upregulated mRNAs from microarray. As a consequence, 25 miRNAs and 76 genes formed 142 miRNA-target gene pairs with an inverse correlation of expression. Among the 422 significantly decreased mRNAs, 33 mRNAs were the predicted targets of the 5 upregulated miRNAs. Similarly, 43 in 350 overexpressed mRNAs and 20 downregulated miRNAs were predicted as targets. According to the above method, a total of 142 significant miRNA-mRNA pairs were predicted, consisting of the 25 differentially expressed miRNAs and 76 mRNAs. The target prediction of 8 miRNAs isn't available in miRWalk databases.

\subsection{Validation of miRNA and mRNA expression profile by RT-qPCR}

To further investigate the credibility of our microarray based results, qRT-PCR was performed in 12 pairs of matched ARM/control fetal rat terminal hindguts. From the differentially expressed miRNAs and mRNAs observed by microarray, 5 miRNAs (Down: rnomiR-1-3p, rno-miR-99b-3p, rno-miR-206-3p, Up: rno-miR-598-3p, rno-miR-3084a-3p) and 5 mRNAs (Down: Fgf16, Wnt16, Cdh15, Up: Trim9, Ccl7) were chosen for RT-PCR validations. Relative expression levels of the selected miRNAs and mRNAs were depicted in Fig. 5A, Fig.

5B. The qRT-PCR results confirmed the accuracy of microarray findings. Compared with controls, rno-miR-598-3p and rno-miR-3084a-3p were significantly increased, while rno-miR-1$3 p$, rno-miR-99b-3p, and rno-miR-206-3p were significantly decreased in the terminal hindgut tissues of ARM fetal rat. Besides, qRT-PCR analysis revealed that the expressions of Trim9, Ccl7 were upregulated, whereas Fgf16, Wnt16, and Cdh15 were downregulated in the terminal hindgut tissues of ARM fetal rat, which is in line with the microarray data, too. Although the 
231 magnitude of changes differed between the two methods, these results demonstrated a high

232 consistency between the microarray and RT-qPCR.

233

234

235

236

237

238

239

240

241

242

243

244

245

246

247

248

249

250

251

\subsection{The regulatory network of miRNAs and target genes}

The miRNA-target gene pairs by Cytoscape software was used to construct the miRNAtarget genes regulatory network. Using the 142 miRNA-target gene pairs, a miRNA-target gene regulatory network was constructed (Fig. 6). In this network, rno-miR-128-3p, rno-miR-200a-3p, rno-miR-30c-2-3p, rno-miR-495, and mo-miR-543-3p, which regulate 15, 12, 13, 15 and 13 targets, respectively, demonstrated the highest connectivities. Whereas Igf1, Fut4, Kcnk2, Ngfr, and Nap112, which were regulated by 8, 6, 4, 4 and 4 miRNAs, respectively, were the mRNAs with the highest connectivities. According to the the network analysis, most of the identified transcripts were connected with several miRNAs, it suggested that multiple miRNA-mRNA interactions combinatorial effect in gene regulation by coexpressed endogenous miRNAs in ARMs.

\section{DISCUSSION}

ARMs have reported to be a multigene genetic disease. Both genetic and environmental factors are involved in the etiopathogenesis of ARMs. The spectrum of ARM phenotypes are ranging from stenotic anus to cloacal malformation, and the etiology of ARMs is still unknown (Wang $C$ et al., 2015). The development of hindgut in the embryonic period is governed by multiple genes in the relevant signaling pathways. A result of genetic change in any stage of the hindgut development may lead to varieties of ARM phenotypes. Recent studies demonstrated that molecular and genetic facors were the contributing roles in its etiology. Only when the 
252

253

254

256

257

258

259

260

261

262

263

264

265

266

267

268

269

270

271

272

cascades of relevant genes expression are well orchestrated, can the normal anorectum develop.

Hence, the further intensive study of ARMs may be benefical to illuminate the etiopathogenesis

of this complex congenital malformation.

Studies of microRNAs (miRNAs) indicated that it is a series of noncoding RNAs with a length of 22 nucleotides, and it has a function of mediating silence and post-transcriptional regulation of gene expression (Ambros V, 2004; Bartel DP, 2004), and involved in many vital processes, including proliferation, cell differentiation, migration and apotosis (Hosako H et al., 2009). The principle of miRNAs is that the miRNA usually interacted with the 3 ' untranslated regions (3'-UTRs) of their targets mRNAs to negatively regulate gene expression at the posttranscriptional level (Saito Y et al., 2009). Up to now, there were more than 2500 human miRNAs recorded in the miRBase (Kozomara A et al., 2011; Kozomara A et al., 2014), and the research showed that more than $60 \%$ of protein-coding transcripts were predicted to be the targets of miRNAs and regulated by miRNAs (Fabian MR et al., 2010). However, there is paucity of literature related to the microRNA expression profiles and their biological effects in ARMs.

In the present study, we evaluated the terminal hindgut expression of miRNAs and mRNAs in both ETU-induced ARM rats and control rats using microarray analysis. We found 33 miRNAs and 772 mRNAs differentially expressed in hindgut tissues between the ARM fetal rats and the controls. GO terms and KEGG pathway analysis for the differentially expressed mRNA further revealed that the miRNA-mRNA contributed a lot to the development of ARMs. 76 potential target genes for the 25 miRNAs were predicted by intersecting three bioinformatics 
273 databases and differentially expressed mRNAs, resulting in 146 miRNA-mRNA pairs. Morever,

274 the expression of several miRNAs and mRNAs were validated by qRT-PCR and the outcomes

275 were highly in accordance with the microarray data, which confirmed the credibility of our

276 microarray data.

277 Among the 33 miRNAs that were differently expressed in the hindgut tissue of ARM fetal rats according to the miRNA microarray data, previously studies have reported that many of these miRNAs (rno-miR-221-3p, rno-miR-200a-3p, rno-miR-1-3p, rno-miR-133b-3p, rno-miR-

133a-3p, rno-miR-486, rno-miR-451-5p) identified in our result were associated with many

disease such as colorectal disease, prostate and pancreatic cancers (Sun K et al., 2011; Qin J et al., 2014; Kneitz B et al., 2014; Sarkar S et al., 2013). Kneitz B et al reported that miR-221 is an oncogenic miRNA which targeted CD117, and it has a function of preventing cell proliferation and migration in endothelial cells (Kneitz $B$ et al., 2014). In this study, we also found that miRNA-200a-3p was significantly up-regulated, Pichler $M$ et al reported that miRNA-200a-3p is able to exert its regulatory effect on EMT (epithelial to mesenchymal transition) and is involved in cancer stem cell properties in colorectal cancer (Pichler M et al., 2014). miRNAs exerted their function of regulating through inhibiting or degrading the translation of its targets. Hence, it is extremely important to identify miRNAs target genes to clarify the development process of ARM. It is well known that the primary cause of ARMs was mainly attributed to the gene expression abnormalities. Gene mutations have been reported to be the most important contributing roles in the pathogenesis of ARMs. The normal development of hindgut will be disturbed by the mutations of the related genes, which thus lead to a series of 
294 ARM phenotypes.

295 Among the 772 mRNAs that showed to be significantly dys-regulated according to 296 microarray datas, many of these mRNAs (Wnt10a, Wnt16, Fgf7, Fgf9, Fgf16, Fgf2, Bmp3, 297 Hoxd1) identified in our result have been reported previously to be associated with the pathogenesis of ARMs. Based on GO analysis of biological processes and pathways, it revealed that the 772 significantly dys-regulated genes to be mainly relate to muscle contraction, muscle organ development, regulation of muscle contraction, skeletal muscle tissue development, response to drug, positive regulation of myotube differentiation, and these cellular events are closely related to extracellular space, myofibril, extracellular matrix, and contractile fiber in

ARM development process. KEGG pathway enrichment analysis revealed that these differentially expressed mRNAs were potentially associated with the cell adhesion molecules (CAMs), ECM-receptor interaction, focal adhesion, PI3K-Akt signaling pathway, and etc. The

PI3K-Akt signaling pathway is activated by many types of cellular stimuli or toxic insults and regulates fundamental cellular functions such as transcription, translation, proliferation, growth, and survival. The binding of growth factors to their receptor tryosine kinase (RTK) or G proteincouple receptors (GPCR) stimulates class Ia and Ib PI3K isoforms, respectively. PI3K-Akt signaling pathway containing many genes including Colla1, Fgf7, Fgf2, Fgf16, Fgf9, Igf7, Prkzc, and etc, while some of those genes are also implicated in the development process of ARMs.

312 These genes linked to the key pathways such as Shh, Fgf, and Wnt/ $\beta$-catenin signaling pathways or were involved in the regulation of their downstream targets such as Bmp4, Fgf10, Gli2, Gli3, and Wnt5a to trigger the abnormal development of anorectum of ARMs. 
Although some miRNAs and their taget mRNAs did not display negative correlation, its regulatory effect maybe concealed by additional regulatory mechanisms or it remains unclear.

Hence, only the miRNAs and their predicted target mRNAs displayed significant correlation with their cognate miRNAs expression were analysed acccording to our algorithm. In the present

study, through detecting the predicted target genes of the differentially expressed miRNAs, we upregulated in the terminal hindgut tissues of ARM fetal rat, and miR-193 inhibited the expression of Hoxd 13 in ARM fetal rat by targeting Hox, while the normal expresion of Hoxd is absolutely essential to the development of the anorectum. 
336 and inhibiting anterior endoderm cell fate (Gambarini AG et al., 1996). Fgfl6 encoded a member

337 of a family of proteins which possessed broad mitogenic and cell survival activities, and were

338 associated with a variety of biological processes such as embryonic development, tumor growth

339 and invasion, cell growth, morphogenesis, tissue repair. In addition, as predicted by

340 bioinformatics databases, Fgf was the potential target gene of rno-miR-221-5p, rno-miR-133b-3p,

341 rno-miR-381-3p, rno-miR-431, and rno-miR-495. The Wifl (Wnt inhibitory factor 1) gene,

342 which mediates the activity of Wnt (Wingless drosophila integration sites) signaling pathway, is

343 a predicted target gene of the rno-miR-451-5p and rno-miR-495 according to TargetScan 7.1.

344 Wif1 is an inhibitory Wnt (Wingless drosophila integration sites) that negatively regulates Wnt

345 signaling. This gene is a member of the Wnt gene family, it plays a key role in the regulation of

346 cell fate and patterning during embryogenesis (Jönsson M et al., 2001). The Wnt was reported to

347 be a crucial signaling pathway and it has a paramount regulatory effect during the development

348 of anorectum. Bambi (BMP and activin membrane-bound inhibitor) gene was significantly

349 upregulated in terminal hindgut tissue of ARM fetal rats in our results, and is a predicted target

350 gene of rno-miR-128-3p which was significantly downregulated. This gene encodes a

351 transmembrane glycoprotein related to the type I receptors of the transforming growth factor-

352 beta (TGF- $\beta$ ) family, whose members play important roles in signal transduction in many

353 pathological processes, the encoded protein may function to limit the signaling range of the

354 TGF- $\beta$ family during early embryogenesis. Our results showed that there existed an obviously

355 negative correlation between the expression of rno-miR-128-3p and Bambi during the 356 development of ARMs. 

rno-miR-221-3p were altered in the terminal hindgut in ARM fetal rats according to our results,

development of anorectum.

It should be noted that because of temporal and spatial expression pattern of mRNAs during

the hindgut development in rat embryos with ETU-induced ARMs, there are some differences in

our study compared with others. And some limitations also existed in our study. In the one hand,

the expression of mRNAs are influenced by multiple factors, both the upstream miRNA and the

neighbour genes can affect the gene expression, and this condition is not taken into consideration

in our work and can be integrated in future research. And the miRNA/mRNA that predicted to

have potential effect to influence the expression of pathogenic genes need further functional experiment for validation.

\section{Conclusion}

In this work, we identified the differentially expressed miRNAs and mRNAs in the terminal 
378 hindgut between ARM fetal rats and normal ones. We strongly suggest that miRNAs are 379 paramount in regulating gene expression in the pathogenesis of ARM. The present work can be 380 regarded as a new perspective and direction for future research on ARMs.

381

382

383

384

385

386

387

388

Data Availability

389

The following information was supplied regarding data availability: The raw data have been

390 uploaded as a Supplemental File.

391

392

393

References

394

Ambros V. 2004. The functions of animal microRNAs. Nature 431(7006): 350-355 Doi

395

10.1038/nature02871.

396

Bai YZ, Chen H, Yuan ZW, Wang W. 2004. Normal and abnormalembryonic development of

the e anorectum in rats. J Pediatr Surg 39(4): 587-590.

398

Bartel DP. 2004. MicroRNAs: genomics, biogenesis, mechanism, and function. Cell 116(2):

399

281-297.

400

Böttcher RT, Niehrs C. 2005. Fibroblast growth factor signaling during early vertebrate 
401

402

403

404

405

406

407

408

409

410

411

412

413

414

415

416

417

418

419

420

421

development. Endocrine Reviews 26(1): 63-77 Doi 10.1210/er.2003-0040.

Endo M, Hayashi A, Ishihara M, Maie M, Nagasaki A, Nishi T, Saeki M. 1999. Analysis of 1992 patients with anorectal malformations over the past two decades in Japan. Steering Committee of Japanese Study Group of Anorectal Anomalies. Journal of Pediatric Surgery 34(3):435-441.

Fabian MR, Sonenberg N, Filipowicz W. 2010. Regulation of mRNA translation and stability by microRNAs. Annual Review of Biochemistry 79:351-379 Doi 10.1146/annurev-biochem060308-103103.

Gambarini AG, Miranda MT, Viviani W, Oyama Júnior S, Kiyota S, Toma IN. 1996. Structure and function of fibroblast growth factor. Brazilian Journal of Medical and Biological Research 29(7): 835-839.

Godwin JG, Ge X, Stephan K, Jurisch A, Tullius SG, Iacomini J. 2010. Identification of a microRNA signature of renal ischemia reperfusion injury. Proceedings of the National Academy of Sciences of the United States of America 107(32): 14339-44 Doi 10.1073/pnas.0912701107.

Hosako H, Martin GS, Barrier M, Chen YA, Ivanov IV, Mirkes PE. 2009. Gene and microRNA expression in p53-deficient day 8.5 mouse embryos. Birth Defects Research. Part A, Clinical and Molecular Teratology 85(6): 546-555 Doi 10.1002/bdra.20565.

Jin S, Wang J, Chen H, Xiang B. 2017. Differential miRNA expression analysis during late stage terminal hindgut development in fetal rats. Journal of Pediatric Surgery 52(9): 1516-1519 Doi 10.1016/j.jpedsurg.2017.02.015.

Jönsson M, Andersson T. 2001. Repression of Wnt-5a impairs- DDR1 phosphorylation and 
422 modifies adhesion and migration of mammary cells. Journal of Cell Science 114(Pt 11): 2043-

4232053.

424 Khanna K, Sharma S, Pabalan N, Singh N, Gupta DK. 2018. A review of genetic factors 425 contributing to the etiopathogenesis of anorectal malformations. Pediatric Surgery International 426 34(1): 9-20 Doi 10.1007/s00383-017-4204-2.

Kneitz B, Krebs M, Kalogirou C, Schubert M, Joniau S, van Poppel H, Lerut E, Kneitz S,

Scholz CJ, Strobel P, Gessler M, Riedmiller H, Spahn M. 2014. Survival in Patients with

High-Risk Prostate Cancer Is Predicted by miR-221, Which Regulates Proliferation, Apoptosis, and Invasion of Prostate Cancer Cells by Inhibiting IRF2 and SOCS3. Cancer Research 74(9): 2591-2603 Doi 10.1158/0008-5472.CAN-13-1606.

Kozomara A, Griffiths-Jones S. 2011. miRBase: integrating microRNA annotation and deepsequencing data. Nucleic Acids Research 39(Database issue): D152-7 Doi 10.1093/nar/gkq1027.

Kozomara A, Griffiths-Jones S. 2014. miRBase: annotating high confidence microRNAs using deep sequencing data. Nucleic Acids Research 42(Database issue ): D68-73 Doi 10.1093/nar/gkt1181.

Kondoh K, Kobayashi K, Nishida H. 2003. Suppression of macho-1-directed muscle fate by FGF and BMP is required for formation of posterior endoderm in ascidian embryos. Development 130(14): 3205-3216.

Levitt MA, Peña A. 2005. Outcomes from the correction of anorectal malformations. Current Opinion in Pediatrics 17(3):394-401.

Lewis BP, Shih IH, Jones-Rhoades MW, Bartel DP, Burge CB. 2013. Prediction of 
443 mammalian microRNA targets. Cell 115(7): 787-98.

444 Mattick JS, Makunin IV. 2006. Non-coding RNA. human molecular genetics 1: R17-29.

445 Pichler M, Ress AL, Winter E, Stiegelbauer V, Karbiener M, Schwarzenbacher

\section{D, Scheideler M, Ivan C, Jahn SW, Kiesslich $\quad$ T, Gerger $\quad$ A, Bauernhofer $\quad$ T, Calin}

447 GA, Hoefler G. 2014. miR-200a regulates epithelial to mesenchymal transitionrelated gene expression and determines prognosis in colorectal cancer patients. British Journal of Cancer 110(6): 1614-1621 Doi 10.1038/bjc.2014.51.

Qin J, Luo M. 2014. MicroRNA-221 promotes colorectal cancer cell invasion and metastasis by targeting RECK. Febs Letters 588(1):99-104 Doi 10.1016/j.febslet.2013.11.014.

Saito Y, Friedman JM, Chihara Y, Egger G, Chuang JC, Liang G. 2009. Epigenetic therapy upregulates the tumor suppressor microRNA-126 and its host gene EGFL17 in human cancer cells. Biochemical and Biophysical Research Communications 379(3): 726-731 Doi10.1016/j.bbrc.2008.12.098.

Sarkar S, Dubaybo H, Ali S, Goncalves P, Kollepara SL, Sethi S, Philip PA, Li Y. 2013. Down-regulation of miR-221 inhibits proliferation of pancreatic cancer cells through upregulation of PTEN, p27(kip1), p57(kip2), and PUMA. American Journal of Cancer Research 3(5): 465-477.

Sun K, Wang W, Zeng JJ, Wu CT, Lei ST, Li GX. 2011. MicroRNA-221 inhibits CDKN1C/p57 expression in human colorectal carcinoma. Acta Pharmacologica Sinica 32(3):375-384 Doi 10.1038/aps.2010.206.

Thum T, Condorelli G. 2015. Long noncoding RNAs and microRNAs in cardiovascular 
464

465

466

467

468

469

470

471

472

473

474

475

476

477

478

479

480

481

482

483

484

pathophysiology. Circulation Research 116(4):751-62 Doi 10.1161/CIRCRESAHA.116.303549

\section{Tsuda T, Iwai N, Deguchi E, Kimura O, Ono S, Furukawa T, Sasaki Y, Fumino S, Kubota}

Y. 2011. PCSK5 and GDF11 expression in the hindgut region of mouse embryos with anorectal

malformations. Eur J Pediatr Surg 21(4):238-241 Doi 10.1055/s-0031-1273691.

Van der Putte SC. 1986. Normal and abnormal development of the anorectum. Journal of

Pediatric Surgery 21(5): 434-440.

Wang C, Li L, Cheng W. 2015. Anorectal malformation: the etiological factors. Pediatric

Surgery International 31(9): 795-804 Doi 10.1007/s00383-015-3685-0.

Wang KC, Chang HY. 2011. Molecular mechanisms of long noncoding RNAs. Molecular Cell 43(6): 904-14 Doi 10.1016/j.molcel.2011.08.018.

\section{Wong EH, Ng CL, Lui VC, So MT, Cherny SS, Sham PC, Tam PK, Garcia-Barceló MM.}

2013. Gene network analysis of candidate Loci for human anorectal malformations. PLoS One 8(8): e69142 Doi 10.1371/journal.pone.0069142.

\section{Figure and Table Legends}

Fig. 1 The flow diagram that describe the process of the microarray and data analysis.

Fig. 2 Unsupervised hierarchical clustering of differentially expressed miRNAs in ARM group.

Red indicates higher expression and green indicates lower expression in hindgut tissue of ARM

fetal rat. White means no expression difference.

Fig. 3 GO and KEGG pathway analysis of differentially expressed miRNAs predicted target genes. (A) GO functional annotation of differentially expressed miRNAs predicted target genes 
485 (Top 10), including biological process, cellular component and molecular function. (B) Predicted 486 target mRNAs of differentially expressed miRNAs enriched in the KEGG pathway scatter plot 487 showing the statistics of pathway enrichment in the ARM group.

488 Fig. 4 GO enrichment and KEGG pathway analysis of differentially expressed mRNAs. (A) Top 48910 pathway enrichment terms for differentially expressed intersection mRNAs, including

490

491

492

493

494

495

496

497

498

499

500

501

502

503

504

505

biological process, cellular component and molecular function. (B) KEGG pathway analysis of the differentially expressed mRNAs was performed. Top 30 pathway enrichment terms for differentially expressed intersection mRNAs.

Fig. 5 qRT-PCR validation of differentially expressed miRNAs and mRNAs. Quantitative reverse transcription was performed to confirm the expression of 5 selected miRNA (A) and 5 mRNA (B).

Fig. 6 The regulatory network between miRNAs and target genes in ARM. The round and square represent the mRNAs and miRNAs, respectively. The purple and yellow colors represent the relatively low and high expression, respectively. The large geometric drawing indicates the more miRNAs or genes interacted with it.

Table. 1 List of differentially expressed miRNAs between ARM and control fetal rats Table.2 Top 20 up- and downregulated mRNAs between ARM and control fetal Rats 
507

508

509

PeerJ reviewing PDF | (2018:05:28325:1:1:CHECK 22 Aug 2018) 


\section{Figure 1}

The flow diagram that describe the process of the microarray and data analysis.

18 Healthy and mature SD rats (Female/male:12/6)

6 pregnant rats (ETU-induced)

3 ARM fetal rats were chosen for microarray analysis

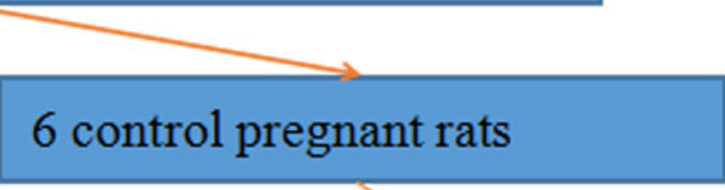

3 control fetal rats were chosen for microarray analysis

RNA extraction \& quantification \&quality control

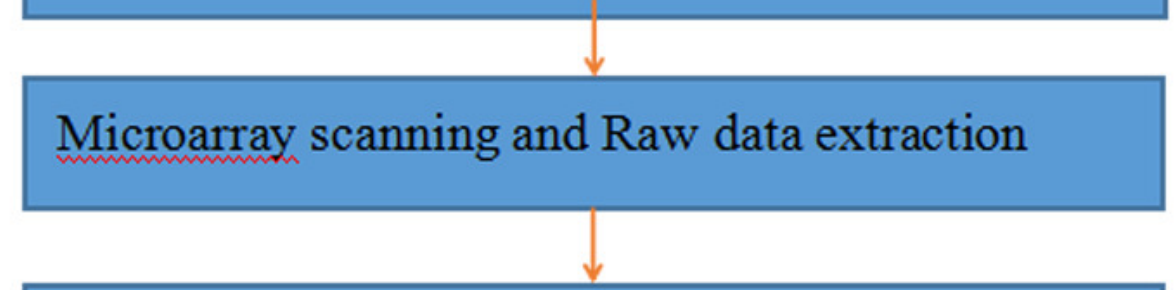

Raw data normalization and probe screening

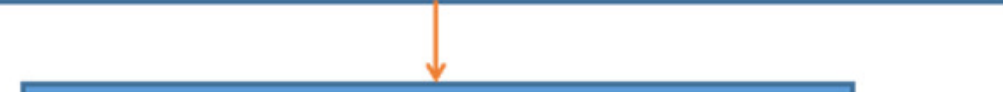

Target gene prediction

GO analysis and pathway analysis

Co-expression network

Further analysis 


\section{Figure 2}

Unsupervised hierarchical clustering of differentially expressed miRNAs in ARM group.

Red indicates higher expression and green indicates lower expression in hindgut tissue of ARM fetal rat. White means no expression difference.

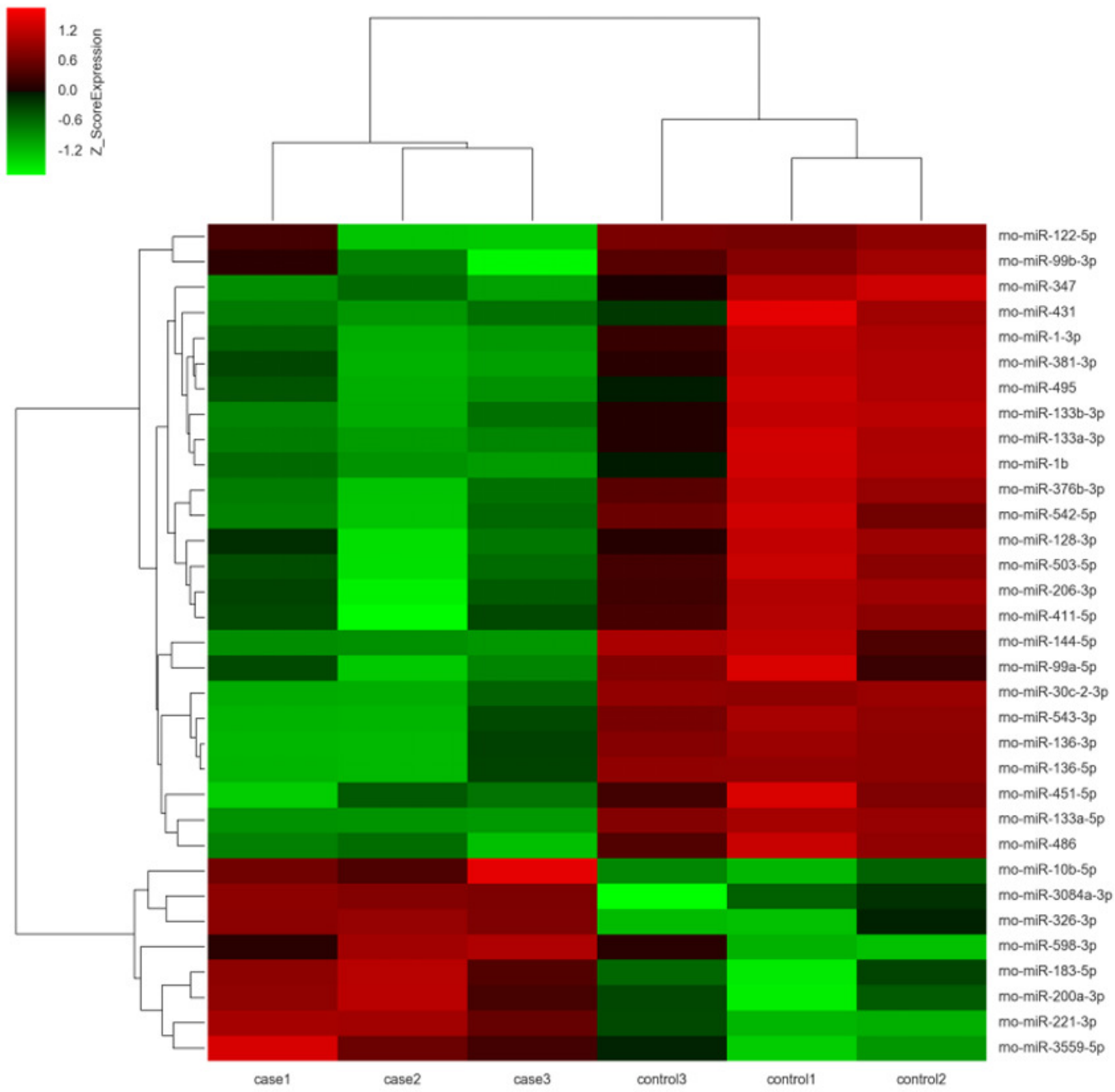




\section{Figure 3}

GO and KEGG pathway analysis of differentially expressed miRNAs predicted target genes.

(A) GO functional annotation of differentially expressed miRNAs predicted target genes (Top 10), including biological process, cellular component and molecular function. (B) Predicted target mRNAs of differentially expressed miRNAs enriched in the KEGG pathway scatter plot showing the statistics of pathway enrichment in the ARM group. 
A

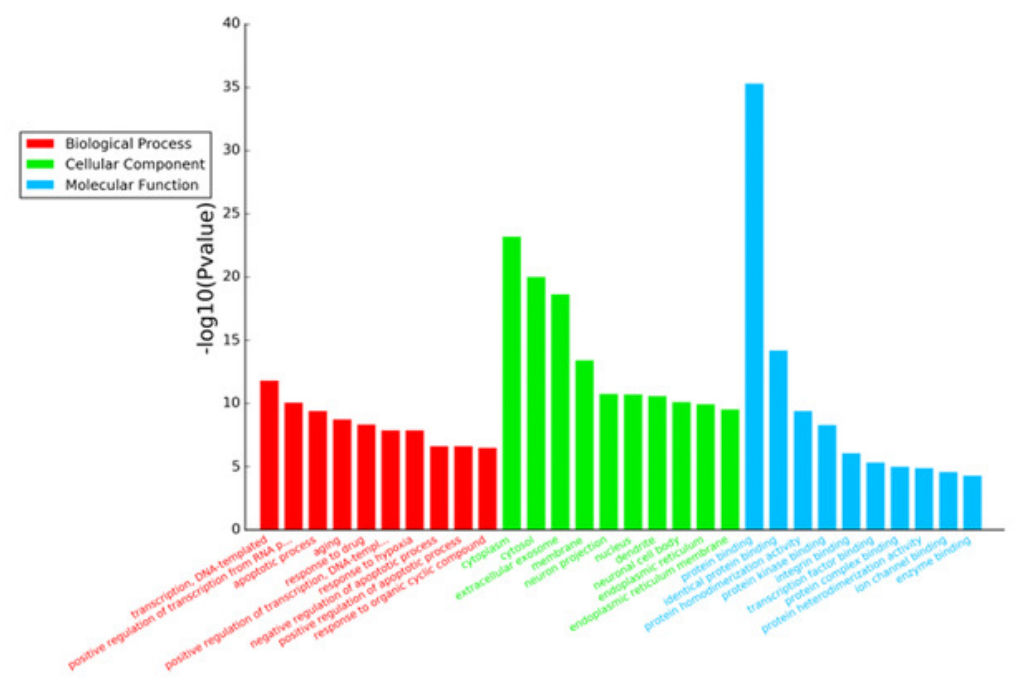

B

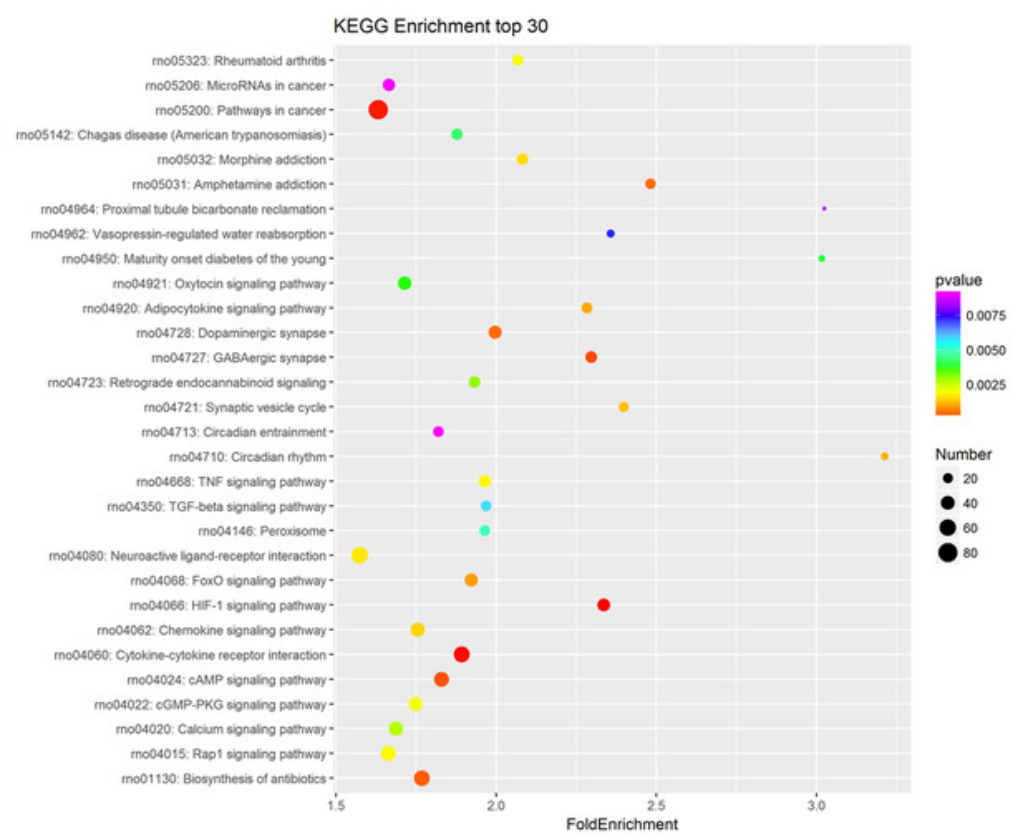


Figure 4

GO enrichment and KEGG pathway analysis of differentially expressed mRNAs.

(A) Top 10 pathway enrichment terms for differentially expressed intersection mRNAs, including biological process, cellular component and molecular function. (B) KEGG pathway analysis of the differentially expressed mRNAs was performed. Top 30 pathway enrichment terms for differentially expressed intersection mRNAs.

A

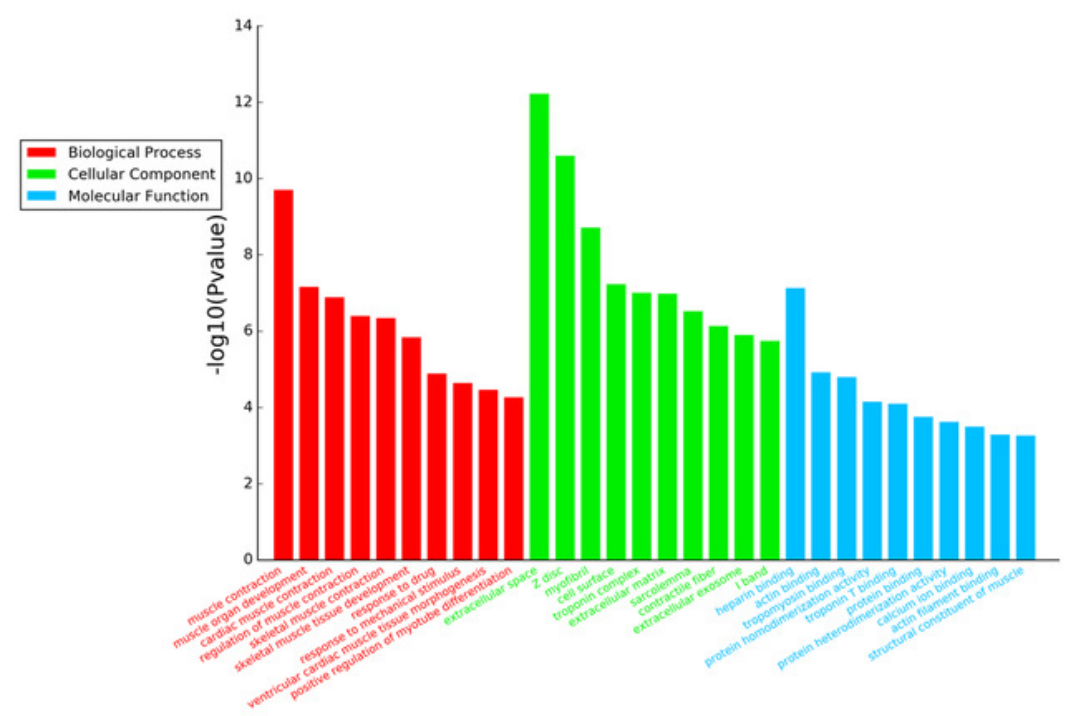

B

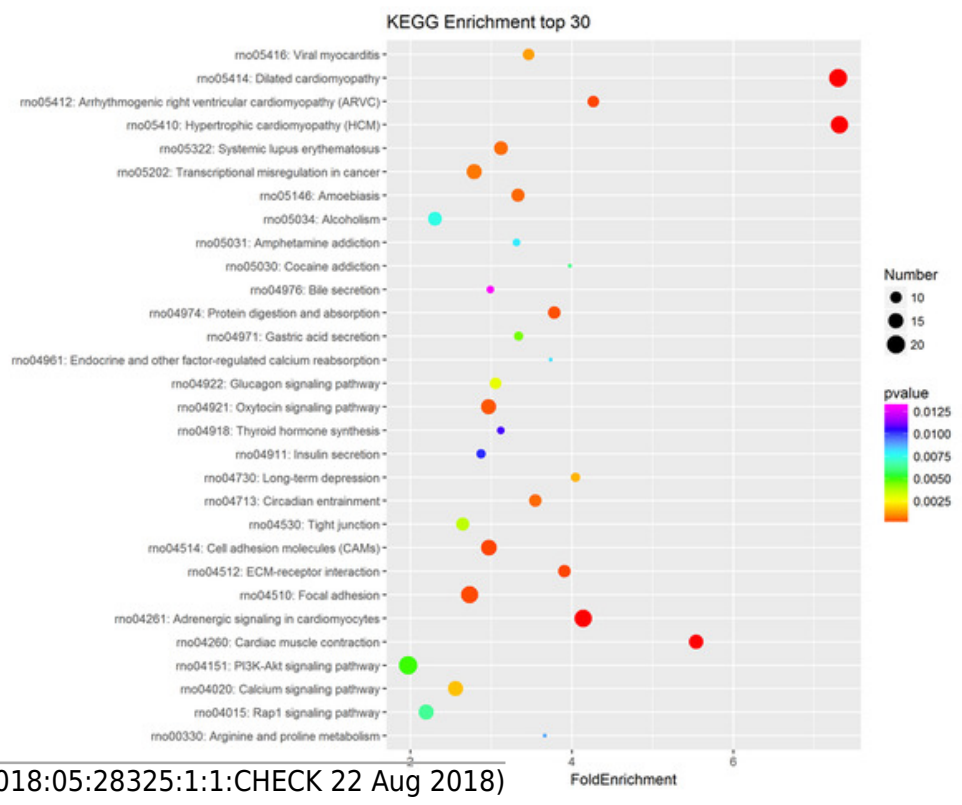




\section{Figure 5}

qRT-PCR validation of differentially expressed miRNAs and mRNAs.

Quantitative reverse transcription was performed to confirm the expression of 5 selected miRNA (A) and 5 mRNA (B). 
A

MiRNA

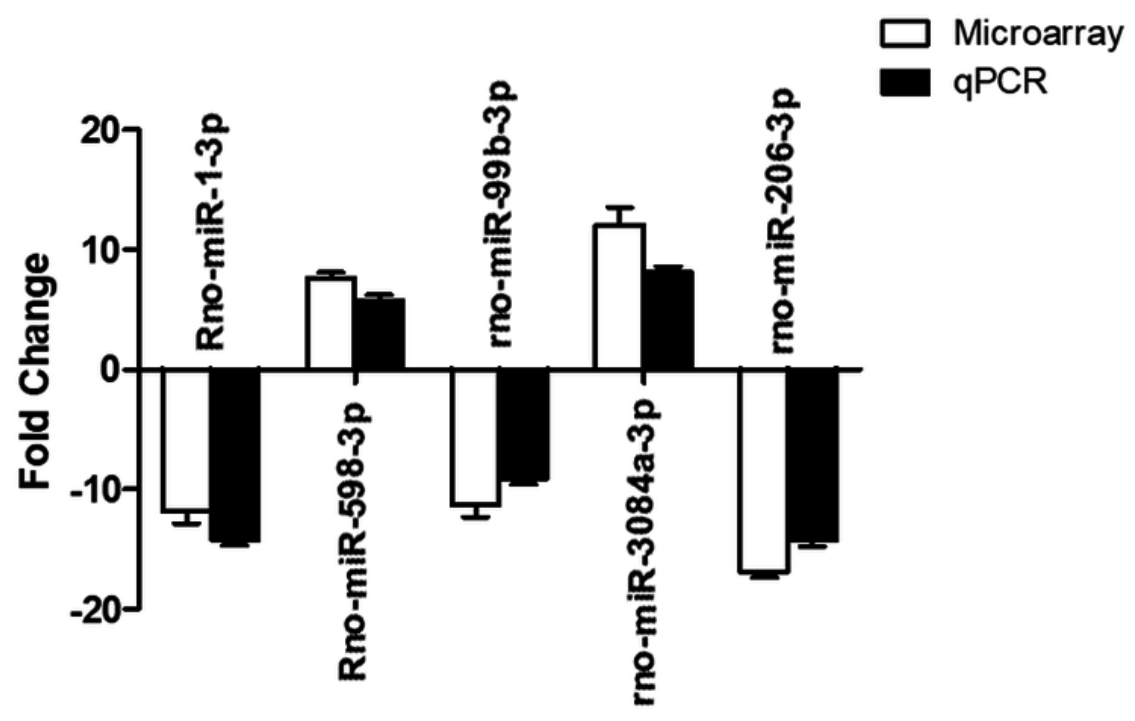

B

mRNA

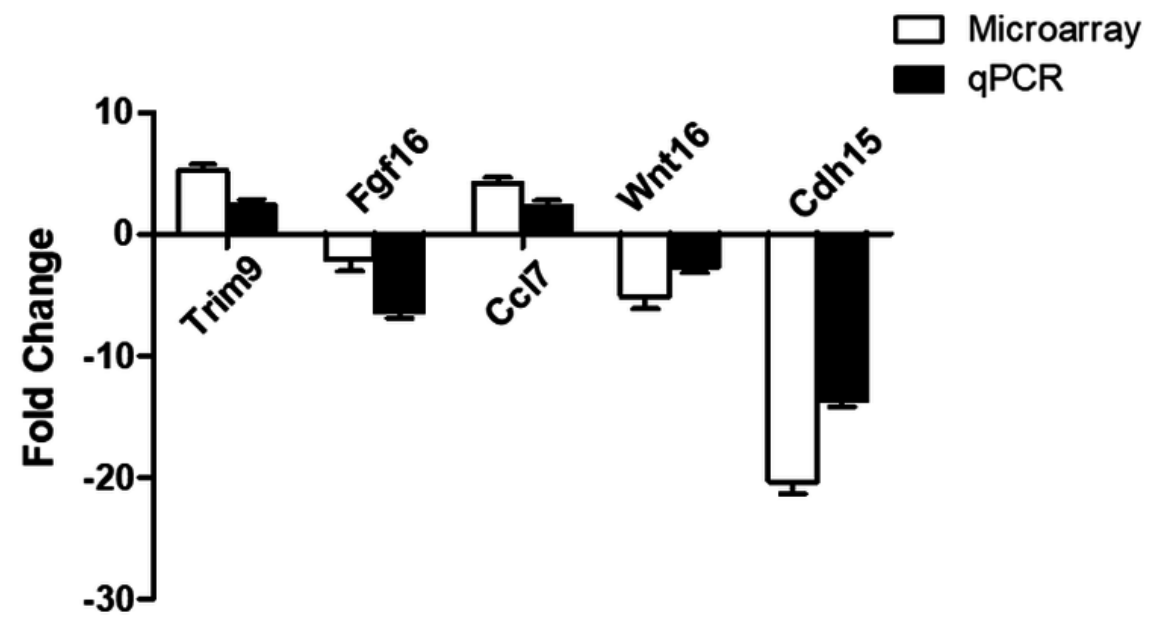




\section{Figure 6}

The regulatory network between miRNAs and target genes in ARM.

The round and square represent the mRNAs and miRNAs, respectively. The purple and yellow colors represent the relatively low and high expression, respectively. The large geometric drawing indicates the more miRNAs or genes interacted with it.

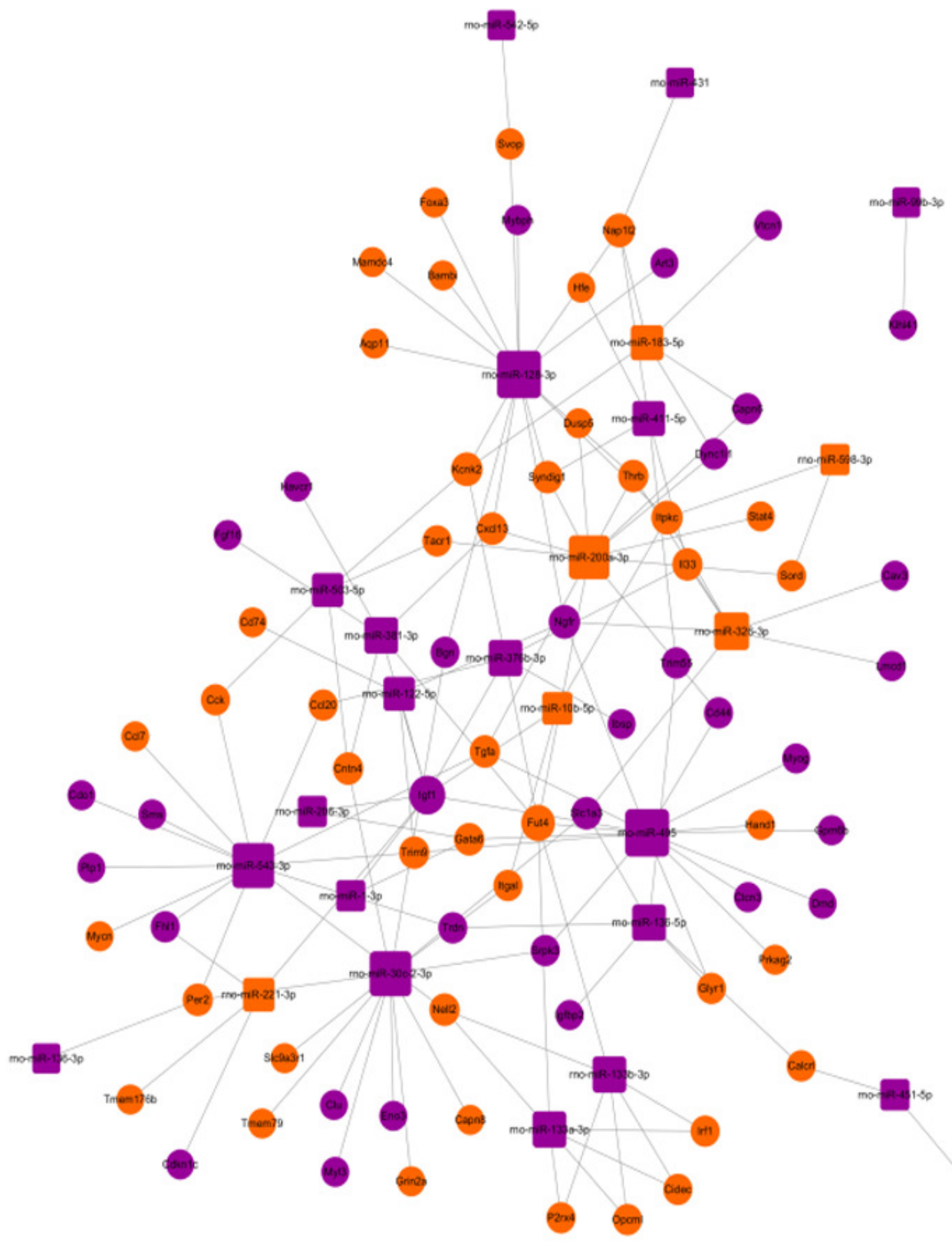




\section{Table $\mathbf{1}$ (on next page)}

List of differentially expressed miRNAs between ARM and normal rats 


\begin{tabular}{|c|c|c|c|c|c|}
\hline Upregulated miRNAs & $P$ value & FC (abs) & Downregulated miRNAs & $P$ value & FC (abs) \\
\hline rno-miR-221-3p & 0.0044 & 2.836 & rno-miR-133a-5p & $1.51 \mathrm{E}-05$ & 128.189 \\
\hline rno-miR-10b-5p & 0.0118 & 2.653 & rno-miR-30c-2-3p & $6.21 \mathrm{E}-04$ & 45.897 \\
\hline rno-miR-326-3p & 0.0129 & 40.909 & rno-miR-144-5p & 0.0024 & 43.322 \\
\hline rno-miR-183-5p & 0.0201 & 2.305 & rno-miR-543-3p & 0.0034 & 67.572 \\
\hline rno-miR-3084a-3p & 0.0259 & 13.513 & rno-miR-136-5p & 0.0037 & 40.949 \\
\hline rno-miR-200a-3p & 0.0266 & 2.153 & rno-miR-136-3p & 0.0041 & 46.235 \\
\hline rno-miR-3559-5p & 0.0352 & 2.116 & rno-miR-376b-3p & 0.0042 & 2.160 \\
\hline \multirow[t]{14}{*}{ rno-miR-598-3p } & 0.0495 & 7.129 & rno-miR-486 & 0.0053 & 3.118 \\
\hline & & & rno-miR-542-5p & 0.0054 & 2.070 \\
\hline & & & rno-miR-1-3p & 0.0093 & 10.819 \\
\hline & & & rno-miR-133a-3p & 0.0143 & 3.407 \\
\hline & & & rno-miR-133b-3p & 0.0146 & 6.663 \\
\hline & & & rno-miR-451-5p & 0.0172 & 2.869 \\
\hline & & & rno-miR-347 & 0.0183 & 2.557 \\
\hline & & & rno-miR-1b & 0.0192 & 2.778 \\
\hline & & & rno-miR-381-3p & 0.0194 & 2.415 \\
\hline & & & rno-miR-503-5p & 0.0199 & 2.719 \\
\hline & & & rno-miR-99a-5p & 0.0209 & 2.147 \\
\hline & & & rno-miR-495 & 0.0248 & 2.758 \\
\hline & & & rno-miR-206-3p & 0.0300 & 17.344 \\
\hline & & & rno-miR-411-5p & 0.0353 & 2.031 \\
\hline
\end{tabular}




\begin{tabular}{cccc}
\hline rno-miR-128-3p & 0.0413 & 2.567 \\
& rno-miR-431 & 0.0424 & 2.378 \\
& rno-miR-122-5p & 0.0475 & 212.683 \\
& rno-miR-99b-3p & 0.0490 & 12.287 \\
\hline
\end{tabular}

1 Table. 1 List of differentially expressed miRNAs between ARM and normal rats 


\section{Table 2(on next page)}

Top 20 up- and downregulated mRNAs between ARM and normal fetal rats. 
1 Table.2 Top 20 up- and downregulated mRNAs between ARM and normal fetal

2 Rats 


\begin{tabular}{|c|c|c|c|c|c|}
\hline Downregulated mRNAs & Fold change & $P$ value & Upregulated mRNAs & Fold change & $P$ value \\
\hline Ky & 87.915 & 0.0178149 & Rpap1 & 40.312 & 0.0187 \\
\hline Fitm1 & 86.903 & 0.0158851 & Alg2 & 21.131 & $6.17 \mathrm{E}-05$ \\
\hline $\mathrm{Co} 19 \mathrm{a} 2$ & 69.592 & 0.0101831 & $\mathrm{P} 2 \mathrm{rx} 4$ & 19.245 & 0.0133 \\
\hline Cited 1 & 66.896 & 0.0132214 & Duoxa2 & 14.719 & 0.0034 \\
\hline Cryab & 59.710 & 0.0125412 & Cyp4v3 & 13.758 & 0.0404 \\
\hline Serpinf1 & 52.046 & 0.0191088 & RT1-A1 & 11.290 & 0.0312 \\
\hline Ablim3 & 50.569 & 0.0230989 & Dhx32 & 11.101 & $9.78 \mathrm{E}-05$ \\
\hline Gatm & 46.246 & 0.0237851 & Fgf9 & 9.546 & 0.0044 \\
\hline LOC685612 & 44.668 & 0.0173458 & Gucy $1 b 3$ & 8.766 & $3.08 \mathrm{E}-04$ \\
\hline Col7al & 43.956 & 0.0096485 & Snrnp35 & 8.671 & 0.0021 \\
\hline Sgcg & 41.283 & 0.0067426 & RGD1562127 & 8.643 & 0.0016 \\
\hline Ttn & 39.483 & 0.0055567 & Kcnk2 & 8.621 & 0.0116 \\
\hline Myoz1 & 37.311 & 0.0189420 & Aldh1b1 & 8.390 & 0.0175 \\
\hline Gfra4 & 33.696 & 0.0098202 & Sntg1 & 8.082 & $8.35 \mathrm{E}-04$ \\
\hline Csrp3 & 32.095 & 0.0056248 & Mogat2 & 7.291 & $5.74 \mathrm{E}-04$ \\
\hline Npepo & 30.003 & 0.0205087 & Slpi & 7.255 & 0.0237 \\
\hline Clec4f & 28.918 & 0.0107149 & Adamdec1 & 6.929 & $3.22 \mathrm{E}-04$ \\
\hline Epha3 & 28.519 & 0.0070704 & Itgal & 6.750 & 0.0342 \\
\hline Mybpc1 & 28.450 & 0.0113402 & Madcam1 & 6.447 & 0.0352 \\
\hline Zbtb16 & 26.716 & 0.0063771 & Tmem151a & 6.351 & 0.0028 \\
\hline
\end{tabular}

